\title{
Implementation of a Weight Loss Program for Latino Outpatients with Severe Mental Illness
}

\author{
Christina Mangurian · Simriti Chaudhry $\cdot$ Lucia Capitelli · Jonathan Amiel • \\ Felicia Rosario • Carlos Jackson • John W. Newcomer • Francine Cournos • \\ Susan Essock · Diane Barrett • Michael Devlin
}

Received: 19 January 2011/ Accepted: 12 March 2012/Published online: 24 March 2012

(C) Springer Science+Business Media, LLC 2012

\begin{abstract}
To determine feasibility of implementation of a weight loss program for overweight Latinos with severe mental illness. In this quasi-experimental study, a 14-week behavioral weight loss course (extended) was implemented at one clinic. A one-time nutrition class (brief) was given at a sister clinic. Implementation feasibility was assessed by consent and participation rates. Weight was followed for 6 months. Consent rates were high [77\% (49/64) extended; $68 \%(39 / 57)$ brief], and $88 \%(43 / 49)$ of extended subjects participated and $88 \%(38 / 43)$ completed follow-up. Weight loss did not differ between groups. A behavioral weight loss course is feasible to implement for this population.
\end{abstract}

Keywords Obesity $\cdot$ Latino - Severe mental illness . Weight-loss program $\cdot$ Metabolic syndrome

C. Mangurian · S. Chaudhry - L. Capitelli · J. Amiel ·

C. Jackson - F. Cournos - S. Essock - D. Barrett - M. Devlin New York State Psychiatric Institute, 1051 Riverside Drive, New York, NY 10019, USA

C. Mangurian · S. Chaudhry $\cdot$ J. Amiel $\cdot$ F. Rosario ·

C. Jackson - F. Cournos - S. Essock - D. Barrett - M. Devlin

Columbia University, 2960 Broadway, New York, NY 10027, USA

C. Mangurian $(\bowtie)$

University of California, San Francisco, CA 94143, USA

e-mail: christina.mangurian@ucsf.edu

J. W. Newcomer

University of Miami, Miller School of Medicine,

Miami, FL 33136, USA

\section{Introduction}

According to the Center for Disease Control, people with severe and persistent mental illness (SMI) lose more than 25 years of potential life compared to the general population, with cardiovascular disease (CVD) being the leading cause of death (Colton and Manderscheid 2006). CVD risk is increased by the metabolic syndrome, which is in turn increased by obesity (National Cholesterol Education Program [NCEP] 2001). The National Health and Nutrition Examination Survey (NHANES) indicates that approximately $25 \%$ of the US adult population has the metabolic syndrome, with Latinos at the greatest risk of any ethnic group (Park et al. 2003). Several studies have found that people with SMI are at high risk for the metabolic syndrome and CVD (Newcomer and Hennekens 2007; Davidson et al. 2001; Lambert et al. 2003). The Clinical Antipsychotic Trials of Intervention Effectiveness (CATIE) Schizophrenia Trial found that the metabolic syndrome was twice as prevalent among individuals with schizophrenia compared to age-matched NHANES general populations (McEvoy et al. 2005). Outpatients with SMI also have higher odds of having obesity, with women at particularly high risk (McEvoy et al. 2005; Allison et al. 2009; Daumit et al. 2003).

Treatment with certain antipsychotic medications can contribute to increased metabolic risk (Allison et al. 1999; Newcomer 2005). Despite several metabolic screening guidelines for people with SMI taking antipsychotic medications (Marder et al. 2004; Parks et al. 2008), actual screening and treatment continues to be alarmingly low (Morrato et al. 2008; Essock et al. 2009; Haupt et al. 2009; Nasrallah et al. 2006). Part of the problem is limited information available to guide mental health clinicians on strategies to lower cardiovascular risk (Amiel et al. 2008). 
The Schizophrenia Patient Outcomes Research Team (PORT) recently reviewed the body of evidence available and recommended that overweight or obese individuals with schizophrenia should be offered a psychosocial weight loss intervention at least 3 months in duration (Dixon et al. 2010)

Unfortunately, there is a paucity of information about weight loss programs for minorities with SMI, particularly Latinos (Cabassa et al. 2010). According to a recent literature review, among weight loss studies reporting racial/ ethnic composition of people with SMI, only $6 \%$ of the subjects were Latino (45/776) and only one case report of 8 subjects included non-English-speaking participants (Cabassa et al. 2010). This lack of data is worrisome since urban Latinos with SMI have an even higher prevalence of obesity and metabolic syndrome than people with SMI in CATIE or among Latinos in general in NHANES (Hellerstein et al. 2007; Kato et al. 2004). Given the substandard health care received by minorities and SMI, and the rapid growth of the US Latino population, this lack of data on a highly vulnerable population is particularly concerning (Smedley et al. 2003; Alegria et al. 2008).

Our group began to address this problem by adapting a weight loss program for Latinos with SMI. Ganguli and colleagues at the University of Pittsburgh implemented a 14-week, behavioral therapy course for overweight outpatients with schizophrenia spectrum disorders; and more subjects in the weight-loss intervention group lost $\geq 5 \%$ of their body weight than controls (Brar et al. 2005). We selected this intervention because it was manualized, inexpensive, and targeted outpatients. This course also met key PORT elements of weight loss interventions (e.g., portion control, goal setting, weigh-ins). These cultural adaptations have been described previously (Mangurian et al. 2009). Briefly, modifications for this urban, Latino SMI population included Spanish translation, incorporation of traditional Dominican food (e.g., plantains, rice and beans); incorporation of culturally-informed exercises activities (e.g., merengue); incorporation of cultural values (e.g., family); and removal of references less relevant for inner city populations of low socio-economic status (e.g., ownership of car). These modifications are consistent with recommended cultural adaptations to weight loss interventions for non-SMI populations (Mier et al. 2009). Although switching antipsychotic medications can be effective for weight reduction in SMI populations (Newcomer et al. 2008; Weiden et al. 2008), a weight loss program was chosen because of data indicating that Latinos with SMI prefer behavioral techniques over medication changes for antipsychotic-induced metabolic problems (Mangurian et al. 2010).

In this quasi-experimental pilot study, we examined the feasibility of implementation of a culturally-modified behavioral intervention for overweight and obese Latino outpatients with SMI. We hypothesized that the course could be feasibly delivered in a community mental health setting. We also characterized weight changes among participants.

\section{Methods}

Setting

This study was conducted at the two outpatient clinics of the Washington Heights Community Service (WHCS). The WHCS clinics are part of the New York State Office of Mental Health and provide mental health services to people with SMI living in Northern Manhattan. The clinics are similar demographically with an ethnic distribution of 75 $\%$ Hispanic, $15 \%$ African American, $9 \%$ Caucasian, and 1 $\%$ Asian (Hellerstein et al. 2007).

\section{Study Subjects}

As part of a quality improvement initiative, all adults attending the WHCS continuing day treatment program (CDTP) were weighed in September 2007. WHCS psychiatrists referred potential study subjects with BMI $\geq 25 \mathrm{~kg} / \mathrm{m}^{2}$ to the research team for possible inclusion in the weight loss program offered at their clinic. Study inclusion criteria were: (1) BMI $\geq 25 \mathrm{~kg} / \mathrm{m}^{2}$, (2) WHCS CDTP patient status, and (3) age $\geq 18$. Exclusion criteria included: (1) inability to provide informed consent or (2) having a health condition preventing light exercise. Subjects received compensation for initial screening (\$10) and each follow-up assessment (\$5), independent of attendance in course.

The mean age of the study population was 60.5 years (SD 13.3y). The population was predominantly female (64 $\%, 51 / 80)$. Regarding race/ethnicity, $85 \%$ (68/80) were Latino, $11 \%$ (9/80) African American, and $4 \%$ (3/80) Caucasian. Most of the Latinos were of Dominican descent $(79 \%, 54 / 68)$. In regard to language capacity, $59 \%(47 / 80)$ were monolingual Spanish-speakers, $24 \%$ (19/80) bilingual, and $18 \%(14 / 80)$ monolingual English-speaking. Forty-one percent (33/80) were never married, $36 \%$ (29/ $80)$ were separated or divorced, $10 \%(8 / 8)$ widowed, and $13 \%(10 / 80)$ married. In regard to education, $40 \%(32 / 80)$ had less than or equal to $8^{\text {th }}$ grade education, $25 \%(20 / 80)$ completed some high school, $16 \%$ (13/80) were high school graduates, $11 \%(9 / 80)$ completed some college, 6 $\%(5 / 80)$ completed college, and $1 \%(1 / 80)$ unknown. Only $6 \%$ had an annual income ranging from $\$ 18,000$ to $\$ 35,999$, with most not knowing their income status $(53 \%$, $42 / 80$ ) or making less than $\$ 18,000$ yearly $(41 \%, 33 / 80)$. 
The majority of the population was disabled $(66 \%, 53 / 80)$ or unemployed (15\%,12/80). Regarding Axis I diagnosis, $61 \%(49 / 80)$ had schizophrenia spectrum disorders, $20 \%$ $(16 / 80)$ had major depressive disorder, $11 \%(9 / 80)$ had bipolar disorder, with the remaining $8 \%(6 / 80)$ having a variety of other diagnosis. The mean baseline BMI was 33.59 (OSD 5.95). In summary, the study population was predominantly Latino of Dominican descent, Spanishspeaking, low-income, single, had less than a high school education, obese and carried a diagnosis of schizophreniaspectrum disorder. There were no differences in these demographic or diagnostic categories between the two comparison groups.

\section{Study Design}

This was a quasi-experimental pilot study in which subjects received different intensity of interventions dependent upon the clinic they attended. Subjects were not randomized to intervention groups for feasibility reasons. One clinic received the "Brief Intervention" and the sister clinic received the "Extended Intervention" as described below. Because of clinic space and programming constraints, subjects in the extended intervention group were divided into three groups of approximately 15 subjects based upon date of consent for participation and language spoken. Two groups ran from March to June 2008; one group was bilingual and the other was only conducted in Spanish. The third bilingual group was conducted from July to September 2008 .

\section{Description of Interventions}

There were two intervention groups: (1) the extended intervention and (2) the brief intervention. The extended intervention was a culturally-modified, behavioral therapy course based on the Ganguli protocol (Brar et al. 2005). The culturally-modified course was taught by a bilingual, registered dietitian. The course was comprised of 20 classes over 14 weeks, using a treatment manual (Mangurian et al. 2007). All participants were provided with a manual in their preferred language. Classes were initially twiceweekly for 60-min (week 1-6) and then once weekly (weeks 7-14). Each class consisted of a weigh-in and an assessment of number of footsteps taken daily since the prior session (the personal pedometer stored daily recordings for up to 1 week). These values were documented on a bulletin board in the classroom. Following these assessments, an instructional phase (e.g., incorporating exercise into daily routine) was conducted. Each class also had an individual review phase and an exercise component (e.g., 15 min walking in place). Healthy and inexpensive snacks were purchased at a local grocer and provided by the instructor at each class, with the cost per serving, serving size, and pertinent nutrition facts discussed. A WHCS staff nurse sat in on the course to be trained to teach it in the future at no additional expense.

The brief intervention was a one-time 45-min nutrition class taught by the WHCS inpatient registered dietician and all subjects were provided with a one-page bilingual handout of information on healthy eating habits. Subjects at both sites received a personal scale (either TANITA BP$630 \mathrm{~W}$ or Healthometer $845 \mathrm{KL}$ ) and a personal pedometer (OMRON HJ-112 Premium Pedometer with Calorie Counter).

\section{Measures}

Because small pilot studies often lack power to detect main effects (Kraemer 2005), we constructed this pilot study to determine the feasibility of implementing a culturallymodified weight loss program for a high-risk Latino population with SMI. As an indicator of feasibility, consent and participation rates were collected for both groups. Participation was defined as attending at least one class in the course. Class attendance and use of pedometers were other indicators of feasibility and acceptability.

We also evaluated the feasibility of collecting secondary outcome measures described below at baseline, 14-, 26-, and 40-weeks. Secondary outcome measures were weight (and calculated BMI), ATP III criteria for the metabolic syndrome (waist circumference, blood pressure, fasting glucose and fasting lipids). Subjects were also asked to complete a health status questionnaire (SF-12) (Ware et al. 1996); and a Client Satisfaction Questionnaire (Attkisson and Zwick 1982; Roberts and Attkisson 1983). For logistic purposes, all outcome measures were collected 2 weeks prior and after the exact follow-up date. Feasibility to collect these secondary outcome measures was determined by the percentage of collection of these variables. Common problems encountered while collecting these measures were described.

At baseline, subjects completed a brief survey, providing basic demographic information. Psychiatric diagnoses and all medications were collected from the medical chart. Because considerable evidence indicates that some antipsychotic medications can worsen obesity and metabolic syndrome parameters (Lieberman et al. 2005; Casey 2005), changes in psychotropic medication prescription during the intervention could impact weight loss. To evaluate this potential confounder, psychotropic medication history was collected at each of the time points and past psychotropic medication history up to 1 year prior was collected from the medical chart. Based on prior research of chronic, nonmedication naive subjects (Allison et al. 1999; Newcomer 2005; Marder et al. 2004; Baptista et al. 2002), 
antipsychotic medications were divided into three types dependent on weight-promoting potential: (1) High (clozapine, olanzapine, chlorpromazine, thioridizine); (2) Medium (risperidone, quetapine, palperidone, perphenazine, loxapine); and (3) low (aripiprazole, ziprasidone, molindone, haloperidol, fluphenazine, pimozide, thiothixine, trifluoperazine). Given the practice of polypharmacy, subjects were divided into four groups: (1) Taking at least one high-weight promoting antipsychotic medication, (2) taking at least one medium-weight promoting antipsychotic medication, (3) taking only a low-weight promoting antipsychotic medication, (4) taking no antipsychotic medication.

\section{Data Analysis}

Chi-Squared analysis was used to determine differences between the two samples including demographic variables, weight gain liability of antipsychotic medication prescribed, and prevalence of ATP-III-defined metabolic syndrome. An independent samples $t$ test was done to determine differences in age, weight and BMI between the two clinic samples. Feasibility of course implementation was characterized by comparing consent and participation rates to those from another weight loss program involving non-Latino subjects with SMI (Kalarchian et al. 2005). Feasibility of collection of secondary outcome measures was also characterized. Chi-Squared analysis was used to examine the relationship between weight loss and intervention program at the two follow-up time points.

Written informed consent was obtained from participants (New York State Psychiatric Institute/Columbia University Department of Psychiatry Institutional Review Board Protocol \#5575). Some of the authors have received grant/research support from pharmaceutical industries. Given that this was an entirely behavioral intervention and that subjects were selected irrespective of their antipsychotic regimen, it is improbable that this support indicates a conflict of interest for this study. All authors certify responsibility for this publication.

\section{Results}

Feasibility of Implementation of the Weight Loss Course

Of the patients served at the extended intervention clinic, $89 \%(64 / 72)$ were eligible to participate. Seventy-two percent (57/79) of the patients served at the brief intervention clinic were eligible to participate. Consent rates were high, with $77 \%(49 / 64)$ of subjects consenting to participate in the extended intervention clinic and $68 \%$ (39/57) at the brief intervention clinic. Eighty-eight percent (43/49) of consenting subjects in the extended intervention participated in the 14 week course, and $95 \%$ (37/39) of consenting subjects participated in the one-time brief intervention. Consent and participation rates for the extended intervention were comparable or higher than another 3-month behavioral weight loss intervention developed for non-Latino SMI populations (73\% consenting; $77 \%$ participating) (Kalarchian et al. 2005). Of extended intervention participants, $68 \%(28 / 43)$ attended over half of the classes, with mean course attendance being 12.71 (SD 4.99) of the 20 classes. Equipment was used regularly as evidenced by $63 \%(27 / 43)$ of extended intervention subjects wearing pedometers at least $50 \%$ of the time during the study.

Secondary outcome measures were collected in $88 \%$ (38/43) of participants in the extended intervention and 78 $\%(29 / 37)$ in the brief intervention at all follow-up time points up to 6 months. These follow-up rates were comparable to the $83 \%$ rate of another 3-month behavioral weight loss intervention developed for non-Latino SMI populations (Kalarchian et al. 2005).

Although it was feasible to collect secondary outcome measures in this population, there were unexpected challenges, including: (1) subject reluctance to have waist circumference measured depending on the person collecting the measure; (2) subjects were reluctant to have labs drawn; (3) staff were reluctant to have increased work load of additional laboratory draws; (4) staff were reluctant to have frequent laboratory screening because of limited primary care resources; and (5) the study mobile scale was stolen in one of the clinics.

\section{Change in Body Weight}

There were no significant differences between the two intervention groups in mean weight or BMI weights at any follow-up time point. There was also no significant difference in the proportion of subjects that lost or maintained weight between the two interventions at all of the followup time points.

Although not statistically significant, there was a mean raw weight loss at 40-weeks in the brief intervention group (from 205.7lbs SD40.0lbs to 202.5lbs SD39.2lbs) that was primarily due to a subpopulation of that group $(24 \%$; $7 / 29)$ who lost $\geq 5 \%$ of their body weight. These subjects were predominantly women $(86 \%, 6 / 7)$, but otherwise there was a range in education, income, language, marital status, medications, and diagnosis. Informal interviews of these subjects revealed that researcher staff regularly checking their weight in the clinic was very helpful. 
Consumer Satisfaction and Self-Reported Health

Of the subjects participating in the extended intervention, the mean score on the Client Satisfaction Questionnaire (CSQ) was 28.6 ( $\mathrm{SD}=2.8$ ). This reflects high satisfaction with the weight loss course since CSQ-8 scores range from 8 to 32 , with higher values indicating higher satisfaction. Subjects' perceptions of their overall health and emotional health were assessed using the global health scale from the SF-12. On a scale that ranges from 0 (poor health) to 4 (excellent) health, subjects generally reported fair to good health and emotional health, with all subjects showing improvements between baseline and follow-up (health + . $21, p=0.06$, emotional health $+0.35, p<0.01)$. The intervention groups did not differ in terms of health or emotional health.

\section{Development of a Maintenance Class}

Because of consumer desire, a weekly maintenance class developed organically in the clinic after the weight-loss course ended. The maintenance course is co-led by a peer educator, with weekly recordings of weights and pedometer readings, and is based on the culturally-modified manual monthly, 6 months after course completion, $60 \%$ (26/ 43 ) of extended intervention subjects attended at least one maintenance class; and $30 \%$ (13/43) attended over five maintenance classes. Two years after course completion, $23 \%(10 / 43)$ of the original participants attend regularly. Half of those subjects (5/10) have lost weight. The course is viewed as a huge success in the clinic and continues to address cultural aspects of Latino foods and community resources such as local farmers' markets.

\section{Study Sample Antipsychotic Medications}

There were significant differences between clinics in terms of weight gain liability of antipsychotic medications prescribed at baseline, with $72 \%$ (31/43) of extended intervention subjects being prescribed at least one medium or high weight-promoting antipsychotic medication compared with $43 \%(16 / 37)$ of the brief intervention subjects $(p<0.004)$.

Medication changes during the study and up to 1 year prior were also evaluated and there were no significant differences. In the extended intervention group, $71 \%$ (27/ 38 ) stayed on the same medication, $11 \%$ (4/38) changed to a medication that could help them lose weight, and $18 \%$ (7/38) switched to a medication that could make them gain weight. In the brief intervention, $72 \%$ (21/29) stayed on the same medications, $17 \%(5 / 29)$ changed to a medication that could help them lose weight, and $10 \%(3 / 29)$ switched to a medication that could make them gain weight over this same time period.

\section{Discussion}

This pilot study provides evidence that a culturally-modified, 14-week behavioral weight loss course for a predominantly Latino SMI population is promising given feasibility of implementation and acceptability to consumers. We know of no other prior study evaluating implementation of a behavioral weight loss treatment program for this high risk, predominantly Spanish-speaking, Latino SMI population.

Feasibility of Implementation of the Culturally-Modified Weight Loss Course

The high consent and participation rates demonstrate that this culturally-modified behavioral weight loss course is feasible to implement in a community setting. High course attendance rates and the development of a maintenance class at the request of the consumers indicate considerable acceptability of the program and a desire by these predominantly Latino consumers to address their weight problems. The feasibility and acceptability of this intervention are particularly important given PORT recommendations for weight loss programs for people with SMI and the lack of culturally-tailored weight-loss programs developed for this high-risk ethnic population with limited English proficiency (Dixon et al. 2010; Cabassa et al. 2010).

Also notably, it was feasible to collect labs in this high risk community outpatient sample. Given the alarmingly low screening rates of SMI outpatients despite national recommendations (Morrato et al. 2008; Essock et al. 2009; Haupt et al. 2009), and the high rates of weight problems among this Latino population $(80 \%, 121 / 151)$, community mental health administrators should strongly consider mandating at least annual metabolic screening in these high-risk SMI populations that they serve.

\section{Efficacy of Culturally-Modified Weight Loss Course}

Despite considerable subject engagement in the course, there was a lack of substantial weight loss as a result of this culturally-modified behavioral weight loss program. There are several possible explanations for this finding. First, the limitation of the quasi-experimental study design resulted in notable prescription differences between the two cohorts with significantly more extended intervention subjects being prescribed at least one medium or high weightpromoting antipsychotic medication (e.g., olanzapine, 
quetiapine) compared those subjects in the brief intervention. These prescription differences may have caused more weight gain in the extended intervention and might explain the disappointing weight loss findings. Even without the study design limitations, small pilot studies often lack power to detect main effects (Kraemer 2005). Given that weight loss was confounded by significant differences in psychotropic medication prescription, a larger, randomized controlled trial should be done to more accurately assess course impact on weight loss. In addition, since this study was conducted with a predominantly Dominican population, future studies should be done in other Latino subpopulations to determine what similarities and differences exist.

We were surprised to find that almost one quarter of the brief intervention subjects who completed follow-up assessments lost $\geq 5 \%$ of their body weight, and maintained this loss for 6 months. These subjects considered frequent weight checks by research staff to play an important role in this weight loss. It is reasonable to consider that a minimal and inexpensive intervention (e.g., receiving a scale) along with frequent monitoring of weight is useful for a subgroup of participants.

\section{Limitations}

The limitations of this pilot study provide useful information for the development of future studies. As described above, the main limitation is related to the quasi-experimental study design which resulted in significant differences in weight gain liability of antipsychotic medications prescribed at baseline between the two groups. Second, there was a bimodal distribution of weight loss, particularly in the brief-intervention group, which made certain statistical analysis (e.g., propensity-score matching) unfeasible. Third, this pilot study made an assumption that orders written in the chart indicated medication adherence, but adherence rates are low in this SMI population (Weiden 2007). Also, adherence may vary in Latino populations dependent on primary language spoken, and this was not controlled for (Gilmer et al. 2009). Adherence is noteworthy because non-adherence to a high weight-promoting medication could lead to weight loss and impact results.

In summary, clinicians should consider behavioral interventions for Latino SMI populations, as this Dominican Latino population was very interested and willing to attend this course. Given the high rate of obesity among this Latino population with SMI and low metabolic screening rates of SMI outpatients in general (Morrato et al. 2008; Essock et al. 2009; Haupt et al. 2009), public health agencies should encourage regular screening and treatment of this high-risk population.
Conflict of interest During this study, Dr. Mangurian received grant/research support from APIRE/Janssen, APIRE/Lilly, and NARSAD. Ms. Chadhry received grant/research support from the Institute of Human Nutrition, Columbia University. Dr. Amiel received grant/research support from APIRE/Janssen. Dr. Newcomer has received grant/research support from The National Institute of Mental Health (NIMH), NARSAD, Bristol-Myers Squibb, Janssen Pharmaceutica, Pfizer, Inc. and Wyeth; he as served as a consultant to AstraZeneca Pharmaceuticals, Bristol-Myers Squibb, BioVail, H. Lundbeck, Janssen Pharmaceutica, Obecure, Otsuka Pharmaceuticals, Pfizer, Inc., Sepracor, Inc., Solvay Pharma, Inc., Vanda Pharmaceutica and Wyeth Pharmaceuticals; he as been a consultant to litigation; he has been a member of Data Safety Monitoring Boards for Dainippon Sumitomo Pharma America, Inc., Organon Pharmaceuticals USA Inc., Schering-Plough/Merck and Vivus, Inc; finally he has received royalties from Compact Clinicals/Jones and Bartlett Publishing for a metabolic screening form. The remaining authors have no interests to disclose.

\section{References}

Alegria, M., Chatterji, P., Wells, K., Cao, Z., Chen, C. N., Takeuchi, D., et al. (2008). Disparity in depression treatment among racial and ethnic minority populations in the United States. Psychiatric Services, 59(11), 1264-1272.

Allison, D. B., Mentore, J. L., Heo, M., Chandler, L. P., Cappelleri, J. C., Infante, M. C., et al. (1999). Antipsychotic-induced weight gain: a comprehensive research synthesis. American Journal of Psychiatry, 156(11), 1686-1696.

Allison, D. B., Newcomer, J. W., Dunn, A. L., Blumenthal, J. A., Fabricatore, A. N., Daumit, G. L., et al. (2009). Obesity among those with mental disorders: a National Institute of Mental Health meeting report. American Journal of Preventive Medicine, 36(4), 341-350.

Amiel, J. M., Mangurian, C. V., Ganguli, R., \& Newcomer, J. W. (2008). Addressing cardiometabolic risk during treatment with antipsychotic medications. Current Opinion in Psychiatry, 21(6), 613-618.

Attkisson, C. C., \& Zwick, R. (1982). The Client Satisfaction Questionnaire: Psychometric properties and correlations with service utilization and psychotherapy outcome. Evaluation and Program Planning, 5(3), 233-237.

Baptista, T., Kin, N. M., Beaulieu, S., \& de Baptista, E. A. (2002). Obesity and related metabolic abnormalities during antipsychotic drug administration: mechanisms, management and research perspectives. Pharmacopsychiatry, 35(6), 205-219.

Brar, J. S., Ganguli, R., Pandina, G., Turkoz, I., Berry, S., \& Mahmoud, R. (2005). Effects of behavioral therapy on weight loss in overweight and obese patients with schizophrenia or schizoaffective disorder. Journal of Clinical Psychiatry, 66(2), 205-212.

Cabassa, L. J., Ezell, J. M., \& Lewis-Fernandez, R. (2010). Lifestyle interventions for adults with serious mental illness: a systematic literature review. Psychiatric Services, 61(8), 774-782.

Casey, D. E. (2005). Metabolic issues and cardiovascular disease in patients with psychiatric disorders. The American Journal of Medicine, 118(Suppl 2), 15S-22S.

Colton, C. W., \& Manderscheid, R. W (2006). Congruencies in increased mortality rates, years of potential life lost, and causes of death among public mental health clients in eight states. Preventing Chronic Disease 3, A42, Retrieved 1/6/2011, from http://www.cdc.gov/pcd/issues/2006/apr/05_0180.htm.

Daumit, G. L., Clark, J. M., Steinwachs, D. M., Graham, C. M., Lehman, A., \& Ford, D. E. (2003). Prevalence and correlates of 
obesity in a community sample of individuals with severe and persistent mental illness. The Journal of Nervous and Mental Disease, 191(12), 799-805.

Davidson, S., Judd, F., Jolley, D., Hocking, B., Thompson, S., \& Hyland, B. (2001). Cardiovascular risk factors for people with mental illness. The Australian and New Zealand Journal of Psychiatry, 35(2), 196-202.

Dixon, L. B., Dickerson, F., Bellack, A. S., Bennett, M., Dickinson, D., \& Goldberg, R. W. (2010). The 2009 schizophrenia PORT psychosocial treatment recommendations and summary statements. Schizophrenia Bulletin, 36(1), 48-70.

Essock, S. M., Covell, N. H., Leckman-Westin, E., Lieberman, J. A., Sederer, L. I., Kealey, E., et al. (2009). Identifying clinically questionable psychotropic prescribing practices for medicaid recipients in New York State. Psychiatric Services, 60(12), $1595-1602$.

Gilmer, T. P., Ojeda, V. D., Barrio, C., Fuentes, D., Garcia, P., Lanouette, N. M., \& Lee, K. C. (2009). Adherence to antipsychotics among Latinos and Asians with schizophrenia and limited English proficiency. Psychiatric Services (Washington D.C.), 60(2), 175-182.

Haupt, D. W., Rosenblatt, L. C., Kim, E., Baker, R. A., Whitehead, R., \& Newcomer, J. W. (2009). Prevalence and predictors of lipid and glucose monitoring in commercially insured patients treated with second-generation antipsychotic agents. The American Journal of Psychiatry, 166(3), 345-353.

Hellerstein, D. J., Almeida, G., Devlin, M. J., Mendelsohn, N., Helfand, S., Dragatsi, D., et al. (2007). Assessing obesity and other related health problems of mentally ill Hispanic patients in an urban outpatient setting. Psychiatric Quarterly, 78(3), 171-181.

Kalarchian, M. A., Marcus, M. D., Levine, M. D., Haas, G. L., Greeno, C. G., Weissfeld, L. A., et al. (2005). Behavioral treatment of obesity in patients taking antipsychotic medications. The Journal of Clinical Psychiatry, 66(8), 1058-1063.

Kato, M. M., Currier, M. B., Gomez, C. M., Hail, L., \& GonzalezBlanco, M. (2004). Prevalence of Metabolic Syndrome in Hispanic and Non-Hispanic Patients with Schizophrenia. Primary Care Companion to the Journal of Clinical Psychiatry, 6(2), 74-77.

Kraemer, H. C. (2005). A simple effect size indicator for two-group comparisons? A comment on $\mathrm{r}$ equivalent. Psychological Methods, 10(4), 413-419.

Lambert, T. J., Velakoulis, D., \& Pantelis, C. (2003). Medical comorbidity in schizophrenia. The Medical Journal of Australia, 178(Suppl), S67-s70.

Lieberman, J. A., Stroup, T. S., McEvoy, J. P., Swartz, M. S., Rosenheck, R. A., Perkins, D. O., et al. (2005). Effectiveness of antipsychotic drugs in patients with chronic schizophrenia. The New England Journal of Medicine, 353(12), 1209-1223.

Mangurian, C., Goss, E., \& Newcomer, J. W. (2010). Metabolic Screening, Prevalence, and Treatment Preferences in Predominantly Hispanic Severely Mentally Ill Inpatients. Psychiatric Services, 61, 1162-1163.

Mangurian, C., Stowe, M., \& Devlin, M. J. (2009). Obesity treatment for urban, low-income Latinos with severe mental illness. Psychiatric Services, 60(8), 1139.

Mangurian, C., Stowe, M., Violante, J., \& Devlinet, M. (2007). Health Eating and Activity in Latinos Treated in the Heights (HEALTH) Course Manual (English). From http://www.columbia. edu/ fc15/ index_files/PDFs/Manual_En.pdf.

Marder, S. R., Essock, S. M., Miller, A. L., Buchanan, R. W., Casey, D. E., Davis, J. M., et al. (2004). Physical health monitoring of patients with schizophrenia. The American Journal of Psychiatry, 161(8), 1334-1349.

McEvoy, J. P., Meyer, J. M., Goff, D. C., Nasrallah, H. A., Davis, S. M., Sullivan, L., et al. (2005). Prevalence of the metabolic syndrome in patients with schizophrenia: baseline results from the Clinical Antipsychotic Trials of Intervention Effectiveness (CATIE) schizophrenia trial and comparison with national estimates from NHANES III. Schizophrenia Research, 80(1), 19-32.

Mier, N., Ory, M. G., \& Medina, A. A. (2009). Anatomy of Culturally Sensitive Interventions Promoting Nutrition and Exercise in Hispanics: A Critical Examination of Existing Literature. Health Promotion Practice, 11(4), 541-554.

Morrato, E. H., Newcomer, J. W., Allen, R. R., \& Valuck, R. J. (2008). Prevalence of baseline serum glucose and lipid testing in users of second-generation antipsychotic drugs: a retrospective, population-based study of Medicaid claims data. The Journal of Clinical Psychiatry, 69(2), 316-322.

Nasrallah, H. A., Meyer, J. M., Goff, D. C., McEvoy, J. P., Davis, S. M., Stroup, T. S., et al. (2006). Low rates of treatment for hypertension, dyslipidemia and diabetes in schizophrenia: data from the CATIE schizophrenia trial sample at baseline. Schizophrenia Research, 86(1-3), 15-22.

National Institute of Health. (2001). Executive Summary of the Third Report of the National Cholesterol Education Program (NCEP) Expert Panel on Detection, Evaluation, and Treatment of High Blood Cholesterol in Adults (Adult Treatment Panel III). The Journal of the American Medical Association, 28, 2486-2497.

Newcomer, J. W. (2005). Second-generation (atypical) antipsychotics and metabolic effects: a comprehensive literature review. CNS Drugs, 19(Suppl 1), 1-93.

Newcomer, J. W., Campos, J. A., Marcus, R. N., Breder, C., Berman, R. M., Kerselaers, W., et al. (2008). A multicenter, randomized, double-blind study of the effects of aripiprazole in overweight subjects with schizophrenia or schizoaffective disorder switched from olanzapine. The Journal of Clinical Psychiatry, 69(7), $1046-1056$.

Newcomer, J. W., \& Hennekens, C. H. (2007). Severe mental illness and risk of cardiovascular disease. The Journal of the American Medical Association, 298(15), 1794-1796.

Park, Y. W., Zhu, S., Palaniappan, L., Heshka, S., Carnethon, M. R., \& Heymsfield, S. B. (2003). The metabolic syndrome: prevalence and associated risk factor findings in the US population from the Third National Health and Nutrition Examination Survey, 1988-1994. Archives of Internal Medicine, 163(4), 427-436.

Parks, J., Radke, A. Q., \& Mazade, N. A. (2008). Measurement of health status for people with serious mental illness. National Association of State Mental Health Program Directors, $16^{\text {th }}$ technical report. Retrieved 1/6/2011, from http://www.nasmhpd. org/general_files/publications/med_directors_pubs/ NASMHPD $\% 20$ Medical $\% 20$ Directors $\% 20$ Health\%20Indicators\%20Report \%2011-19-08.pdf.

Roberts, R. E., \& Attkisson, C. C. (1983). Assessing client satisfaction among Hispanics. Evaluation and Program Planning, 6(3-4), 401-413.

Smedley, B. D., Stith, A. Y., \& Nelson, A. R. (2003). Unequal treatment: Confronting racial and ethnic disparities in health care. Washington, DC: The National Academy Press.

Ware, J., Jr, Kosinski, M., \& Keller, S. D. (1996). A 12-Item ShortForm Health Survey: construction of scales and preliminary tests of reliability and validity. Medical Care, 34(3), 220-233.

Weiden, P. J. (2007). Understanding and addressing adherence issues in schizophrenia: from theory to practice. The Journal of Clinical Psychiatry, 68(Suppl 14), 14-19.

Weiden, P. J., Newcomer, J. W., Loebel, A. D., Yang, R., \& Lebovitz, H. E. (2008). Long-term changes in weight and plasma lipids during maintenance treatment with ziprasidone. Neuropsychopharmacology, 33(5), 985-994. 